\title{
Desarrollo de las capacidades empresariales: influencia en el crecimiento económico de las MiPyMEs mexicanas
}

\section{Development of business capabilities: influence on the economic growth of Mexican MSMEs}

\author{
Erika Dolores Ruiz' \\ Tecnológico Nacional de México \\ erika.dr@tierrablanca.tecnm.mx \\ Julio Fernando Salazar Gómez ${ }^{* 2}$ \\ Tecnológico Nacional de México \\ juliof.sg@tierrablanca.tecnm.mx \\ Ibis Rafael Huerta Mora \\ Tecnológico Nacional de México \\ i.huerta@tierrablanca.tecnm.mx \\ María de Jesús Valdivia Rivera ${ }^{3}$ \\ Tecnológico Nacional de México \\ maria.vr@tierrablanca.tecnm.mx \\ Manuel Hernández Cárdenas ${ }^{4}$ \\ Tecnológico Nacional de México \\ manuel.hernandez@itstb.edu.mx
}

* Autor corresponsal.

1 Tecnológico Nacional de México, División de Ingeniería en Administración, Prolongación Avenida Veracruz $S / N$, esquina héroes de pue bla, colonia Pemex. Tierra Blanca, Veracruz, MÉXICO.

2 Tecnológico Nacional de México, División de Ingeniería en Sistemas Computacionales, Prolongación Avenida Veracruz S/N, esquina héroes de puebla, colonia Pemex. Tierra Blanca, Veracruz, MÉXICO

3 Tecnológico Nacional de México, División de Ingeniería en Administración, Prolongación Avenida Veracruz $S / N$, esquina héroes de puebla, colonia Pemex. Tierra Blanca, Veracruz, MÉXICO

4 Tecnológico Nacional de México, División de Contador Público, Prolongación Avenida Veracruz $S / N$, esquina héroes de puebla, colonia Pemex. Tierra Blanca, Veracruz, MÉXICO.

INFORMACIÓN ARTÍCULO

Recibido: 18 de Agosto 2021

Aceptado: 12 de Noviembre 2021

Palabras Claves:

Capacidades empresariales

Crecimiento económico

MiPyMEs

Madurez 


\begin{abstract}
The objective is to determine the development of business capacities and their influence on the economic growth of companies in Tierra Blanca, Veracruz, Mexico, to compare them with other studies and see if the conditions are met. Quantitative, correlational, cross-sectional study. The R Studio software version 1.4 .1103 was used. Obtaining a statistical quantitative analysis, without drawing conclusions (inferences), being a regional study, with Micro, Small and Medium Enterprises (MSMEs) from 11 to 50 employees and with a time of permanence equal to or greater than seven years in the market. A semi developed level of the capacities of the organizations was found, with a significant weak influence on economic growth, inviting an analysis of other variables, there is no similarity with the literature. The study is carried out in the Tierra Blanca region, Veracruz, because it is the area of greatest influence of the Tecnológico Nacional de México (TecNM), Tierra Blanca campus and it is where it seeks to generate greater business development. The subject is relatively new, the literature focuses individually on capacities, but not on carrying out a global study of all of them, evaluating the level of development and its influence on the economic growth of itself.
\end{abstract}

\section{ARTICLE INFO}

Received: 18 August 2021

Accepted: 12 November 2021
Keywords:

Business capabilities

Economic growth

MSMES

Maturity

\section{INTRODUCCIÓN}

La realidad que hoy aqueja al mundo es muy drástica, las empresas se encuentran en un escenario crítico en donde no solo luchan con la competencia, sino que también se ven afectados por factores externos como lo es la inflación, aspectos políticos, sociales y culturales, además de un fenómeno epidemiológico (Covid-19) que restringe a la humanidad a una vida convencional, lo que obliga a estas organizaciones a establecer nuevas estrategias que sean más eficaces para lograr sus objetivos organizacionales. No obstante, para poder cumplir con ellos es de gran valor identificar cuáles son las capacidades empresariales que poseen las Micro, Pequeñas y Medianas Empresas (MiPyMEs) y en qué nivel de desarrollo se encuentran actualmente. En este contexto, la teoría económica de la empresa se centra en específico en señalar que el éxito recae en el conocimiento (Ornelas, 2017), en otras palabras, la fórmula correcta es un conocimiento sólido acompañado del desarrollo de capacidades fundamentales que cada empresa posee y las habilidades que ejecute el administrador al interior de sus entidades económicas.

Por lo tanto, las capacidades empresariales son un elemento indispensable en el desarrollo organizacional que contribuye a promover la competitividad de las empresas, a generar una gestión de mayor impacto en los indicadores institucionales, así como establecer líneas de acción oportunas para enfrentar los fenómenos económicos en los que están inmersos. Cabe hacer mención que, en empresas de gran tamaño el desarrollo de las capacidades no es tan complejo como se presenta en las MiPyMEs, debido a que las condiciones y recursos que poseen estas compañías facilitan la operatividad y, por ende, la proyección de estrategias a nivel macro les permite competir con grandes modelos de negocios. Entonces, partiendo del escenario donde las MiPyMEs, a pesar de ser un segmento empresarial que tienen grandes aportes al producto interno bruto y es gran generador de empleo, no están exentas de enfrentar enormes retos día a día a nivel administrativo y operativo que frecuentemente las obliga a retroceder en su evolución y crecimiento económico de sí misma.

El fenómeno descrito no solo ocurre en las MiPyMEs mexicanas, sino que afecta a todas las empresas de este tipo en América Latina, razón por la que se han realizado estudios que evalúan este conglomerado de empresas con la finalidad de determinar los alcances de las políticas públicas que propician la competitividad empresarial. Las investigaciones demuestran que existe una amplia oferta de servicios para el desarrollo de las organizaciones, aunado a un marco institucional que promueve la innovación y el financiamiento que merece ser reconocido (CEPAL, 2019). Sin embargo, la fortaleza que pueden llegar a desarrollar estas empresas requiere de trabajo interno, porque las habilidades y destrezas de cada directivo juegan un rol muy importante para crear una amalgama que combine todos esos beneficios 
que se generan a través de políticas públicas y la operatividad interna de cada organización. Este fenómeno no es ajeno en la región, hoy por hoy estas empresas enfrentan serios problemas en lo que respecta a su operatividad, por lo que, sin duda alguna, es una situación preocupante que requiere de atención para conservar estas fuentes de empleo que son primordiales para la población.

Por ello, esta investigación tiene como objetivo determinar el desarrollo de las capacidades empresariales y su influencia en el crecimiento económico de las MiPyMEs en Tierra Blanca, Veracruz, México. Lo que permitirá definir el nivel de madurez de las capacidades empresariales en lo individual y lo colectivo para poder conocer en profundidad cuál es el estatus que guardan, y con ello generar acuerdos en relación con sus necesidades, recursos y estrategias. De esta forma, mejoran su operatividad y obtienen un mayor crecimiento económico, con acceso a migrar a un modelo de negocio de mayor magnitud, creando un valor agregado en sus productos, así como a nivel unidad de negocio.

Lo anterior corresponde a las necesidades que emergen del modelo educativo que persigue el Tecnológico Nacional de México, Campus Tierra Blanca, siendo esta región la de mayor influencia. Por lo tanto, sobre la base de los argumentos antes mencionados, se plantea la siguiente interrogante ¿Cuál es el nivel de desarrollo de las capacidades empresariales y su influencia en el crecimiento económico que presentan las MiPyMEs en la región de Tierra Blanca, Veracruz?

El presente documento se encuentra estructurado por seis apartados, en primer lugar, se realiza la introducción, seguido de la explicación detallada de la metodología que se utilizó para el desarrollo del estudio, posterior, una revisión de la literatura con datos actuales en materia de las variables que se estudian, las cuales muestran hallazgos sólidos con relación a las capacidades empresariales y el crecimiento económico en MiPyMEs. Como parte medular se plantean los resultados obtenidos y se analizan para plasmar las conclusiones pertinentes, cerrando con las declaraciones de conflictos de intereses. De manera general, los resultados que se resaltan marcan un fenómeno generalizado bastante interesante que demuestra que el desarrollo de las capacidades empresariales tiene efectos en el crecimiento económico de las empresas estudiadas, pero también estos resultados dejan un preámbulo para estudiar otras variables que influyen en el crecimiento económico de las
MiPyMEs y que contribuyen a fortalecer las capacidades empresariales.

\section{DISEÑO METODOLÓGICO}

Para el desarrollo de este estudio se recurrió a un estudio cuantitativo, no experimental, de alcance correlacional-explicativo, transversal, con una población compuesta de 308 empresas tomadas del Sistema de Información Empresarial Mexicano (SIEM) (2019), la muestra es probabilística estratificada, con un nivel de confianza del 95\% y un margen de error del $5 \%$, teniendo un total de 172 empresas tomadas aleatoriamente de los sectores comerciales, industriales, y de servicios.

Se establecieron criterios de exclusión por parte de los investigadores definidos, especialmente por encontrarse constituidas de 11 a 50 empleados y tener más de siete años en el mercado, aspectos que se consideran en una MiPyME con mayor desarrollo. El objetivo de la investigación es determinar las capacidades empresariales y su influencia en el crecimiento económico de las empresas en la región de Tierra Blanca, Veracruz. La aplicación del instrumento se realizó mediante encuesta directa en los meses de marzo-abril de 2020. Cabe mencionar que, en México la pandemia empezó a hacer estragos a partir del día 20 de marzo de 2020, sin embargo, las empresas en la región empezaron a cerrar obligadas por las disposiciones de la autoridad en la semana cuatro de abril del año en curso, quedando solo abiertas las empresas de primera necesidad Esto generó la oportunidad de poder terminar con la aplicación de los instrumentos.

El tratamiento de las variables se efectuó por medio de la segregación por dimensiones, siendo capacidades laborales, comerciales, financieras, tecnológicas, productivas y logística para la variable independiente capacidades empresariales, las cuales fueron evaluadas con un instrumento que se compone de 30 ítems, propuesto por De la Cruz, Morales y Carrasco (2006), estructurado por una escala de Likert en donde el valor 0 es nunca y 4 siempre. La metodología empleada para medir las capacidades empresariales sugiere una recopilación de información de la operatividad de las empresas, se sistematiza la información de las capacidades identificadas, se miden, se analiza la información recopilada para generar un modelo donde el puntaje mínimo es 0 el máximo es 20. 
Por su parte, para la variable dependiente crecimiento económico de las MiPyMEs, las dimensiones que se definieron son índice de ventas y crecimiento en capital, para la recolección de datos se utilizó un instrumento compuesto por 17 ítems donde 0 es nunca y 4 siempre, dicho instrumento se genera porcentualmente debido a datos estimados. Esta decisión se tomó según la postura de las empresas participantes, al no estar en la disposición de proporcionar datos tan específicos, las razones recaen en el sigilo de la información financiera por seguridad. El procesamiento de los datos de cada instrumento se realizó por medio del software R Studio versión 1.4.1103. Las técnicas de análisis de datos estadísticos por medio de un análisis de regresión y correlaciones de las variables, apoyados además en la visualización de datos y análisis de los escenarios.

Derivado de lo anterior y para efectos de este estudio, las capacidades empresariales han sido clasificadas en capacidades laborales, comerciales, financieras, tecnológicas, de producción y logísticas, se decide esta categorización de acuerdo con el nivel de operaciones y recursos con los que normalmente cuenta una empresa catalogada como MiPyME.

\section{REVISIÓN DE LITERATURA}

Hablar de capacidad empresarial es muy complejo dada la magnitud que puede tener cada uno de los países, con relación al aparato empresarial que cada uno posee, sin embargo, para fines de este estudio se centra en las capacidades empresariales de las MiPyMEs. En esta dinámica, las capacidades empresariales se definen por ser habilidades que tienen las empresas y que contribuyen a mejorar la gestión de los recursos. Castrillón y Urrego (2020) y Acosta-Medina, Plata-Cómez, Puentes-Garzón y Torres-Berreto (2019) se concentran en conceptualizar la capacidad empresarial como un conjunto de rutinas específicas que se crean a través de una serie de actividades que generan valor para la operatividad organizacional, aunado a que en conjunto se caracterizan por establecer relaciones sinérgicas con todos los recursos, permeando hacia el logro de metas y objetivos organizacionales que, en otro contexto individualizado, dificultaría el logro esperado.

Por ello, considerando la importancia que tienen las capacidades en el cumplimiento de los objetivos organizacionales, la planeación de estas re4 presenta un elemento fundamental en la toma de decisiones. Autores como Becerra y Herrera (2018) han demostrado que las capacidades son decisivas en cualquier nivel, razón por la cual como investigadores se coincide con el autor en el aspecto de que si son estructuradas correctamente pueden responder a necesidades fluctuantes que comúnmente se suscitan en el mercado, por lo tanto, la planeación debe ser definida, correcta, precisa y oportuna.

Estudios muestran un análisis crítico de las capacidades empresariales, donde la organización al fungir como un sistema abierto, se ve generalmente influenciada de manera intermitente por una diversidad de factores de contingencia que se mezclan y que afecta la visión de sus directivos al momento de dirigir la organización (Ponce-Espinosa, Espinoza, Ríos-Zaruma y Tapia, 2017), entonces, el desarrollo de las capacidades puede llegar a crear una ventaja competitiva reflejada en un significativo crecimiento (López-Salazar, Molina-Sánchez y Gómez-Hernández, 2018).

Lo interesante radica en el análisis de cada una de ellas y establecer el funcionamiento individual, para lo cual es necesario entender cómo las capacidades empresariales contribuyen al mejoramiento de las empresas. Esparza y Martínez (2019), resaltan hallazgos direccionados a una relación muy estrecha entre la capacidad productiva y el proceso, viéndose reflejado en la calidad y en el cumplimiento de las expectativas de cada cliente. Este aspecto puede afectar al índice de ventas.

En tanto, Cajigas, Ramírez y Ramírez (2019) confirman los resultados antes mencionados al encontrar que la capacidad de producción define los recursos y capacidades internas que han de desarrollar todos los indicadores pertinentes para definir correctamente la participación que tendrá en el mercado, evitando errores que anteriormente se cometían por la falta de conocimiento o de información. En esta línea, Donawa y Morales (2018) demuestran que los directivos de las empresas son medulares si poseen conocimiento para generar cambios radicales que transformen situaciones de manera coordinada y estratégica, lo que conlleva a posicionar a la organización dentro de los mejores lugares, volviéndola más competitiva y con oportunidad de mantener esa posición a largo plazo.

Marín (2017), se centró en el estudio de la capacidad de absorción, y destaca específicamente la exigencia que deben tener las empresas para 
disponer de recursos e invertir en conocimiento, partiendo de la premisa que el factor humano es uno de los elementos más importantes para las organizaciones, por ello, estos deben de contar con las competencias idóneas para asimilar el conocimiento de forma que se vea una real transformación de los procesos que posee la unidad de negocio.

En esta dinámica, las capacidades competitivas son creadoras de ventaja competitiva y van a permitir una diferenciación con los competidores (Mojica y Martínez, 2017). Asimismo, es importante cómo se vinculan directamente con el capital humano para crear una mayor ventaja competitiva, lo cual es congruente porque al poseer conocimiento permite una participación activa enfocada en el desarrollo del capital humano, creando una sinergia positiva en pro de una mayor competitividad empresarial, propiciando el aumento en las ventas, utilidades y la absorción del mercado.

En consecuencia, Nuryakin, Didiek y Budi (2018) llegaron a la conclusión de que existe una relación poco significativa entre el rendimiento que tiene una empresa y la capacidad relacional, pero que genera un efecto positivo en la generación de valor y coincide con Mojica y Martínez (2017) al comprobar que las capacidades de una organización contribuyen a incrementar el conocimiento de mercado. Sin duda alguna, las capacidades de adaptación proporcionan herramientas para generar todos esos cambios que demandan las empresas acordes a las necesidades que se van presentando en un mercado tan volátil.

En esta línea, Acevedo-Gelves y Albornoz-Arias (2019) descubrieron que las capacidades tecnológicas vienen a fortalecer a las organizaciones por medio de la sistematización de los procesos, por lo que la aplicación de tecnologías propicia un mejoramiento en las estrategias. En tanto, Hernández, Marulanda y López (2014) e Islas y Palomo (2018), convergen en que las capacidades tecnológicas se han convertido en una herramienta operacional indispensable para la gestión de información confiable que permite a las empresas generar una mayor competitividad. Al respecto, la capacidad logística se relaciona con la tecnológica, porque depende de un cúmulo de elementos que interactúan de manera determinante en su nomenclatura, que se identifican como factores internos, generales e incontrolables (Gamboa-Poveda, Armijo-Borja, Pluas-Salazar y Tovar-Arcos, 2019).
Por consiguiente, la relación que exista entre la empresa y su tamaño es relevante debido a que es una característica primordial para el crecimiento de la empresa, tomando en cuenta que cuando se habla de crecimiento, y el desarrollo que podría en un momento referirse al nivel de madurez de las capacidades empresariales que posee una empresa, hace alusión al nivel de desarrollo máximo que tiene cada una de sus capacidades empresariales y que trabajan en sinergia hacia un escalonamiento mayor, donde se visualiza una evolución empresarial (Melgarejo, Ciro y Simón Elorz, 2019).

Aunado a lo anterior, la variable crecimiento económico se entiende como el crecimiento que tiene una organización en relación con su productividad que puede ser visualizada desde dos puntos: el índice de ventas, es decir, la rentabilidad que posee, lo cual se traslapa a mostrar una mayor seguridad $y$, por otra parte, se encuentra el crecimiento en capital que integra la solvencia que se refleja en la estabilidad de los recursos financieros de la unidad de negocio (Delfín y Acosta, 2016).

Cabe señalar que, la mayor parte de la literatura se centra en definir y determinar el crecimiento económico de las empresas desde una perspectiva global. En este sentido, debe entenderse como el conglomerado de empresas que conforman al país, y cómo estas están aportando al desarrollo económico local, regional y nacional, pero también cómo posiciona a nivel macro al país, en comparación con otros países. El término crecimiento económico también puede conceptualizarse como un proceso que debe ser medido a través de la diferencia de mediciones (León-Mendoza, 2019), que pueden ser el nivel que ha alcanzado la empresa en el tiempo, midiendo indicadores como el volumen de ventas, capitales de mercado y volumen en ventas.

No obstante, Juárez (2018) aborda en su investigación la influencia que tienen los activos, siendo determinantes en el crecimiento de las organizaciones, generando una fórmula basada en la mezcla que existe entre industria, empresa y tamaño. Por lo tanto, partiendo de esta perspectiva, y según la conceptualización que maneja Juárez (2018), aunado al criterio de los investigadores, se toma como crecimiento económico empresarial aquella evolución que puede tener una empresa en sus indicadores económicos y que la hace trascender. En este caso, el índice de ventas y crecimiento en capital son dos elementos de suma importancia para lograr la potencialización de una entidad económica, por lo que el índice de ventas 
permite a la organización lograr una mayor captación de clientes, movilidad de los inventarios, mayores ingresos, mayor liquidez, entre otras. Por parte del capital se crea una mayor fortaleza de la empresa, proyectando una mejor solidez e independencia financiera, en pos de una empresa competitiva y rentable.

Por consiguiente, al realizar la revisión de la literatura y el diseño metodológico se plantea como supuesto de investigación que las capacidades empresariales aumentan el crecimiento económico de las MiPyMEs.

\section{RESULTADOS}

Para realizar la evaluación de las capacidades empresariales se realizó, en primer lugar, la categorización. Para ello, fue necesario conceptualizarlas y comprender en detalle qué es lo que se va a evaluar en cada una (ver Figura 1), reflexionando que se toma como base el instrumento validado por De la Cruz y cols. (2006). Se procedió a identificar a través de un diagnóstico previo cada uno de los indicadores que los autores manejan de forma individual en cada capacidad, esto con la finalidad de verificar que cuentan con las características que se requieren para el estudio, con el objeto de facilitar la aplicación del instrumento.

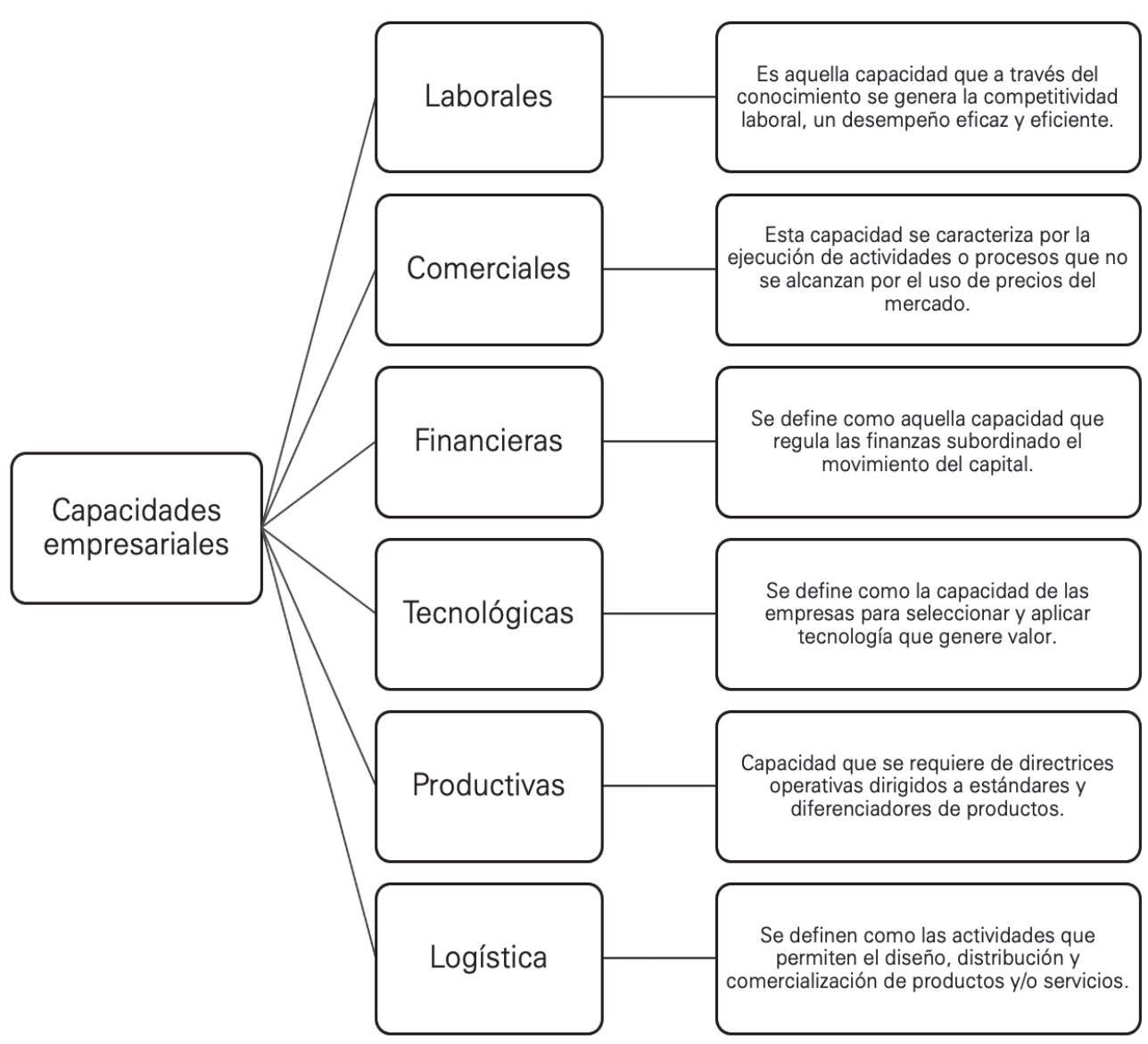

Figura 1. Categorización de las capacidades empresariales. Fuente: elaboración propia.

Una vez identificadas las capacidades empresariales y elegida la muestra, se procedió a la aplicación del instrumento, los resultados que se encontraron se organizaron por sector empresarial 6 (comercial, industrial y de servicios), porque es de interés poder compararlos. Los hallazgos fueron los siguientes: el sector comercial muestra sus capacidades empresariales semi-desarrolladas, sin 
embargo, el sector industrial se destacó por presentar capacidad laboral y financiera desarrollada, en estado semi-desarrollado tiene la capacidad comercial, tecnológica, logística y productiva, en esta dinámica el sector servicios arrojó como capacidad desarrollada la comercial y laboral; no obstante, la capacidad logística se encuentra en un nivel poco desarrollado y, en consecuencia, la capacidad productiva, financiera y tecnológica están semi-desarrolladas (ver Tabla 1). Estos resultados demuestran que, de los tres sectores, el sector comercial es el que se encuentra en nivel más bajo con relación a los otros dos, en los cuales es importante mencionar que el sector industrial resulta ser el mejor evaluado, pues el sector servicios adolece de la capacidad logística (ver Tabla 2).

Tabla 1. Evaluación de las capacidades empresariales

\begin{tabular}{|c|c|c|c|}
\hline \multicolumn{3}{|c|}{ CONCENTRADO DE LA EVALAUCIÓN DE LAS CAPACIDADES EMPRESARIALES } \\
\hline Capacidad & Sector comercial & Sector industrial & Sector servicios \\
\hline Capacidad comercial & 77.12 & 79.37 & 83.75 \\
Capacidad tecnológica & 76.12 & 76.56 & 76.1 \\
Capacidad laboral & 76.5 & 83.12 & 91.5 \\
Capacidad financiera & 76.87 & 81.25 & 75.5 \\
Capacidad logística & 79.12 & 79.65 & 54.25 \\
Capacidad productiva & 76.5 & 77.8 & 77.85 \\
\hline
\end{tabular}

Fuente: elaboración propia.

Tabla 2. Escala de medición propuesta por De la Cruz y cols. (2006).

\begin{tabular}{|l|l|}
\hline & Desarrollada \\
\hline & Semi-Desarrollada \\
\hline & Poco Desarrollada \\
\hline & No Desarrollada \\
\hline & Nula \\
\hline
\end{tabular}

Fuente: elaboración propia.

Se realizó análisis estadístico descriptivo del comportamiento de las empresas comerciales, empresas industriales y empresas de servicio, al evaluar el comportamiento de las variables promedio del crecimiento económico contra el promedio de la capacidad empresarial en cada uno de los sectores. Empleando el software R Studio se consiguió el promedio de una muestra de 172 em- presas pertenecientes a los sectores comerciales, industriales y de servicios, elegidas aleatoriamente. En la Tabla 3 se muestra el comportamiento promedio de evaluación de cada sector con las variables de respuesta, es decir, capacidad empresarial al enfrentarse con el crecimiento económico, lo que permite contrastar las variables objeto de estudio.

Tabla 3. Comportamiento de las capacidades empresariales y crecimiento económico de las empresas participantes (promedios versus desviaciones estándar).

\begin{tabular}{lccc}
\hline \multicolumn{1}{c}{ Variables } & Empresas de Servicio & Empresas Comerciales & Empresa Industrial \\
\hline $\begin{array}{l}\text { Promedio de desarrollo } \\
\text { económico }\end{array}$ & $2.4500 \pm 0.5524$ & $2.6750 \pm 0.4743$ & $2.188 \pm 0.655$ \\
$\begin{array}{l}\text { Promedio de capacidad em- } \\
\text { presarial }\end{array}$ & $3.2500 \pm 0.4935$ & $3.5750 \pm 0.5006$ & $3.688 \pm 0.602$ \\
\hline
\end{tabular}

Fuente: elaboración propia. 
Los resultados encontrados revelan una relación clara al afirmar que los sectores que poseen menor desarrollo en sus capacidades tienden a tener un menor crecimiento económico. En este sentido, la correlación que arroja en el sector comercial es de -0.273 con un valor de $P=0.089$, para el sector servicios la correlación es de -0.235 con un valor de $P=0.144$ y para el sector industrial la correlación es de 0.158 con un valor de $P=0.558$, lo que indica que en los tres sectores la relación de las variables capacidades empresariales frente al crecimiento económico presentan una diferencia significativa al emplear un valor de $p=0.05$.

Se puede observar el comportamiento de la regresión lineal en función de cada uno de los sectores que se estudiaron. Con respecto al sector comercial, la tendencia es decreciente al tener mayor capacidad empresarial con corto crecimiento económico, sin embargo, al tener una capacidad empresarial menor, también lo es el crecimiento (Figura 2). Se demuestra que no tiene un control de estas variables porque los puntos de dispersión se encuentran alejados del comportamiento lineal. Como se puede visualizar, existe una correlación negativa entre las variables objeto de estudio, por lo tanto, es importante analizar otras variables que podrían afectar en mayor medida el crecimiento económico de las empresas objeto de estudio.

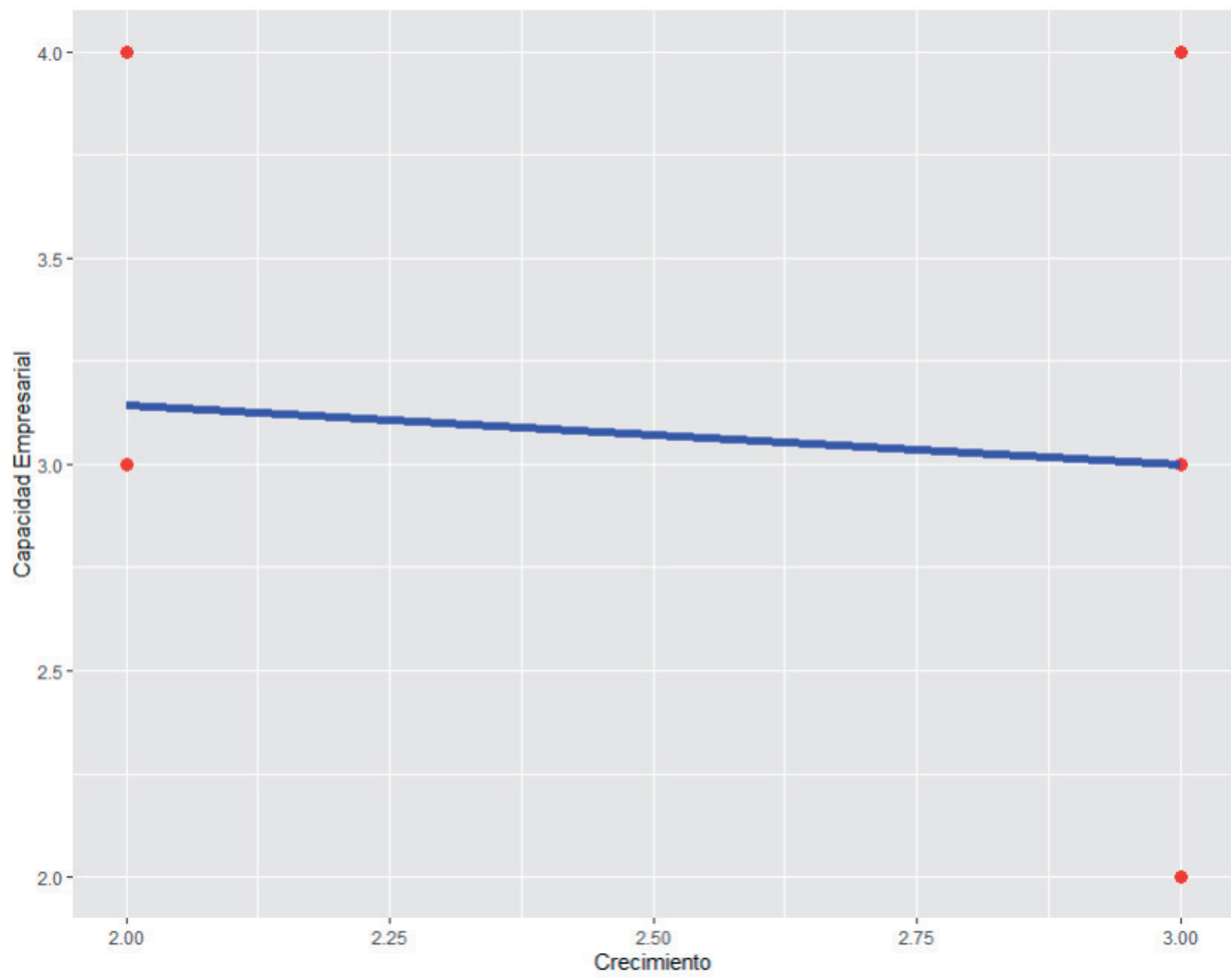

Figura 2. Capacidades empresariales versus crecimiento económico en el sector comercial. Fuente: elaboración propia. 
En la Figura 3 al comparar el sector empresarial industrial con una regresión lineal, se visualiza que las variables estudiadas se comportan estables, es decir, que existe un equilibrio entre la capacidad empresarial y el crecimiento económico, destacando que ambas se encuentran en el mismo marco de crecimiento, lo que, desde la perspectiva teórica, es acorde a una escala de crecimiento constante. Sin embargo, se demuestra que no tiene un control de estas variables porque los puntos de dispersión se encuentran alejados del comportamiento lineal. Como es observable, el contexto que presentan las variables difiere con respecto al sector comercial (Figura 2), porque la correlación que se presenta es de 0.158 siendo positiva baja. Esto implica que las empresas participantes están realizando un uso óptimo de sus capacidades que determina la relación existente.

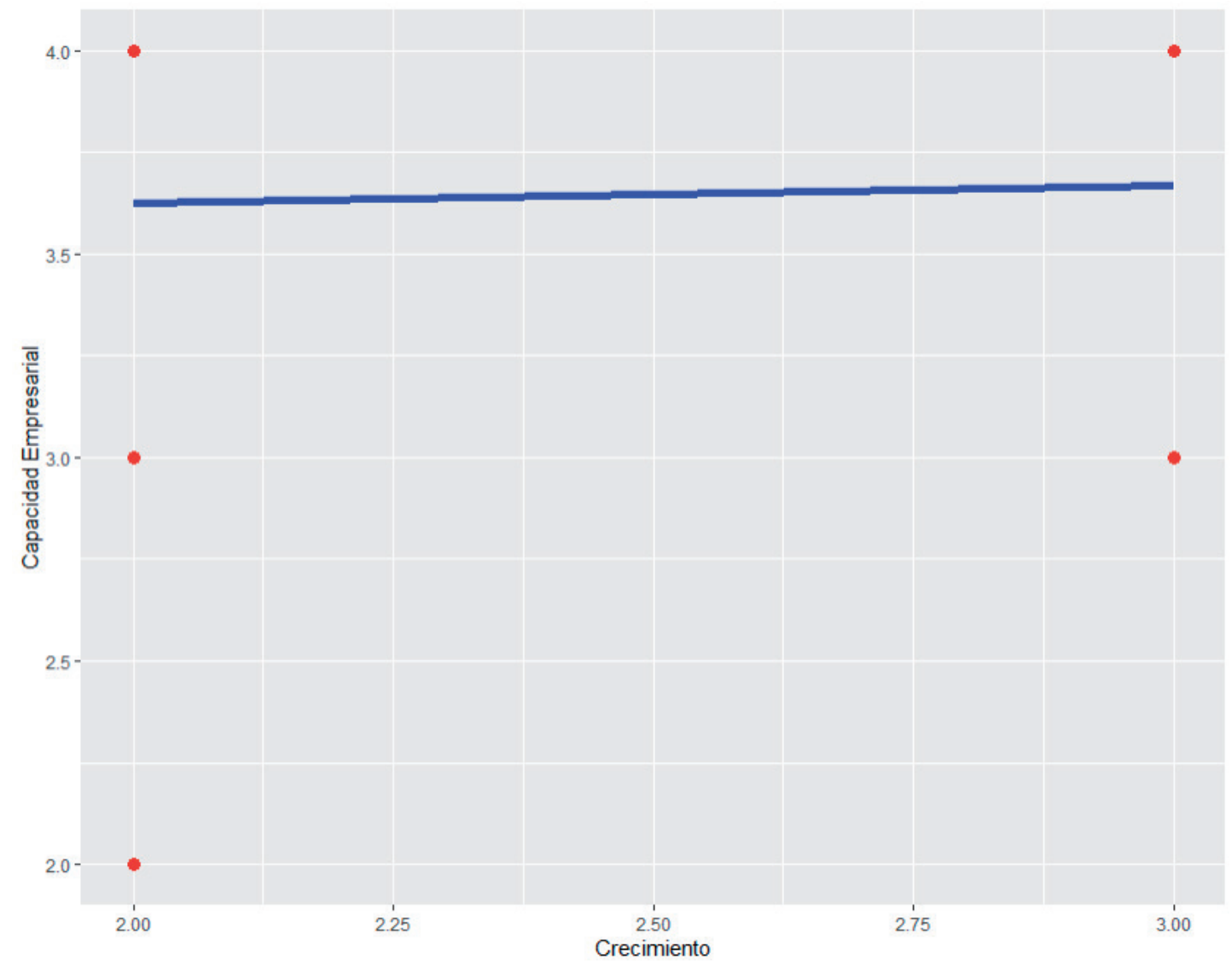

Figura 3. Capacidades empresariales versus crecimiento económico en el sector industrial.

Esta dinámica con relación al sector servicios, muestra que la tendencia es decreciente al tener mayor capacidad empresarial con corto crecimiento económico, sin embargo, al tener una capacidad empresarial menor el crecimiento es menor (ver Figura 4). Se demuestra que no tiene un control de estas variables porque los puntos de dispersión se encuentran alejados del comporta- miento lineal. Se visualiza un fenómeno muy distinto en comparación con los sectores comercial e industrial, por lo que se obtiene una correlación negativa de -0.235. Por tal motivo, se destaca que de los tres sectores que se estudian, el sector industrial es el que está realizando un mayor aprovechamiento de sus capacidades, potencializándolo hacia el crecimiento económico sostenible. 


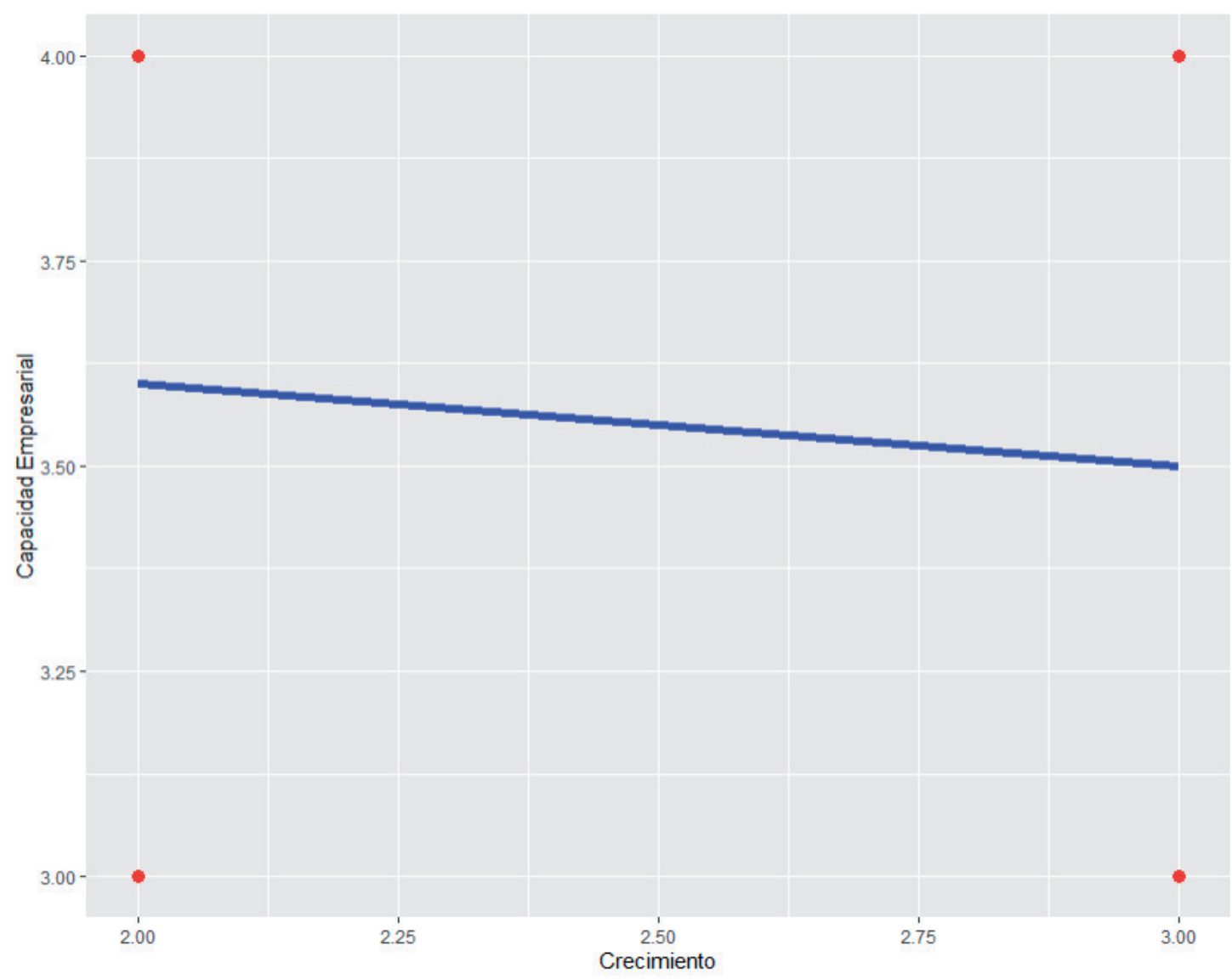

Figura 4. Capacidades empresariales versus crecimiento económico en el sector servicios. Fuente: elaboración propia.

\section{DISCUSIÓN}

Desde los resultados antes presentados, se argumenta que en el sector empresarial objeto de estudio y a partir de la revisión de literatura, se exhibe un esquema bastante sólido al ser empresas que tienen la característica de tener más de siete años en el mercado, porque les permite tener un amplio conocimiento de este, y por obvias razones de sus necesidades operativas. No obstante, en sus capacidades empresariales el resultado de la evaluación las cataloga en un nivel semi-desarrollado, lo que ha ocasionado que su crecimiento económico no sea muy significativo, al encontrar un crecimiento económico calificado como regular e invita a cuestionarse qué es lo que está sucediendo o no se está generando un buen uso de sus capacidades.
Al analizar los estudios de Castrillón y Urrego (2020) e Islas y Palomo (2018), se concluye que el nivel de desarrollo de las capacidades empresariales representa un factor que influye directamente en la productividad y competitividad empresarial, y sus resultados son contundentes en el crecimiento económico de las empresas. En tanto, Nuryakin y cols. (2018) encontraron un efecto significante entre las capacidades y el rendimiento empresarial, por lo que se puede afirmar que hay similitudes en los hallazgos, y es posible considerar que el nivel de desarrollo que tengan las capacidades empresariales de una organización sí influye en el crecimiento económico.

Es importante verificar si las estrategias que se están implementando para alcanzar un crecimiento económico elevado, están siendo las idóneas o definitivamente no han generado una 
planeación estratégica adecuada. El escenario que se plasma es positivo al contar la región con un aparato empresarial MiPyME sólido, definido y estructurado, no obstante, deja un vacío al denotar un crecimiento económico poco relevante, lo que obliga a realizar investigaciones en otras variables que pueden estar interfiriendo en el logro de un desarrollo económico sostenible y efectivo, aunado a que es visible que poseen capacidades con un nivel de desarrollo óptimo, sin embargo, no hacen uso de ellas de manera correcta, lo cual se refleja en resultados relacionados con el crecimiento económico de sí mismas.

\section{CONCLUSIONES}

En general, este estudio se realizó con empresas de los tres sectores empresariales donde el Tecnológico Nacional de México, campus Tierra Blanca, tiene mayor influencia y donde parte de la visión que se tiene es lograr el desarrollo económico y tecnológico de la región. Por ello, se planteó determinar la madurez de las capacidades empresariales de las MiPyMEs y su influencia en el crecimiento económico. Los resultados obtenidos dan respuesta a la pregunta de investigación al demostrar una influencia significativa entre las variables objeto de estudio, encontrando una madurez de las capacidades empresariales semi-desarrolladas.

Se esperaba comprobar una influencia altamente significativa. Los hallazgos llevan a generar reflexión y comprensión de que existen más factores que pueden, en un momento dado, ser determinantes en el éxito o fracaso de una organización, por lo que se determina que el desarrollo de las capacidades empresariales solo es un factor que puede influir en el crecimiento económico de una empresa, pero no es el factor determinante que favorezca el crecimiento económico de las unidades de negocio, rechazándose la hipótesis de investigación.

En este contexto, las capacidades empresariales son un factor que favorece el aumento del crecimiento económico de las MiPyMEs, porque se comprobó que la relación que presentan las variables tiene un nivel de significancia débil. Lo anterior, conlleva a realizar un mayor análisis al interior de las empresas para determinar por qué los resultados no son los esperados, puesto que otros estudios demuestran que el nivel de desarrollo que presentan las capacidades empresaria- les está directamente relacionado con su evolución empresarial.

\section{CONSIDERACIONES FINALES}

Motivado en los resultados se demuestran que, capacidades empresariales y crecimiento económico tienen una significancia débil, quedan abiertas diversas variables que valen la pena estudiar con la finalidad de lograr un mejor crecimiento económico de las MiPyMes, tomando en cuenta que son un segmento de mucha importancia para la economía de la región y del país, al proporcionar un gran aporte del producto interno bruto.

Si se considera que este aporte al producto interno bruto es una de las principales generadoras de empleo en los países de América Latina, sería de gran trascendencia replicar el estudio en otros contextos para ver similitudes y una completa transferencia del conocimiento para alcanzar el desarrollo del aparato empresarial de las MiPyMEs que son determinantes en la generación de empleo y crecimiento económico de sí mismas y de la región.

Por último, es importante destacar que el muestreo se realizó a principios del año 2020, en un contexto que podría definirse como normal, donde los efectos de la pandemia covid-19 aún no hacían grandes estragos en México, por ello los resultados que se obtuvieron no se encuentran influenciados al $100 \%$ por este fenómeno epidemiológico, sin embargo, existe un interés de volver a evaluar a las empresas participantes, para determinar cómo han contribuido sus capacidades empresariales en el manejo de sus empresas en tiempos de pandemia.

Estos resultados serán tomados como base para la generación de proyectos integradores, lo que permitirá la inclusión de alumnos en materia de investigación y generar propuestas que ayuden a potencializar sus capacidades empresariales y mejorar su posición en el mercado.

\section{Agradecimientos}

Se agradece al Tecnológico Nacional de México, campus Tierra Blanca por el apoyo y financiamiento de la investigación. Así como el reconocimiento e interés que mostraron las empresas participantes al contestar objetivamente los instrumentos. 


\section{REFERENCIAS}

Acosta-Medina, J. K., Plata-Gómez, K. R., Puentes-Garzón, D. E., y Torres-Barreto, M. L. (2019). Influencia de los recursos y capacidades en los resultados financieros y en la competitividad empresarial: una revisión de la literatura. I+D Revista de Investigaciones, 13(1), 147-157.

Acevedo-Gelves, L. K., y Albornoz-Arias, N. (2019). Revisión teórica de las capacidades dinámicas empresariales. Pensamiento \& Gestión, 46, 262-283.

Becerra, F. M., y Herrera, R. M. M. (2018). Planeación de capacidades operativas asociadas a la rentabilidad en empresas prestadoras de servicios. Un enfoque de dinámica de sistemas. Tecnura, 22(57), 32-43.

Castrillón, J. J. y Urrego, D. A. (2020). Modelo para la medición del nivel de madurez de capacidades empresariales para la productividad y competitividad, CRL, 1-13.

Cajigas, M., Ramírez, E. y Ramírez, D. (2019). Capacidad de producción y sostenibilidad en empresas nuevas. Revista Espacios, 40(43), $15-28$

CEPAL, (2019). Índice de Políticas PYME: América Latina y el Caribe 2019 Políticas para PYMEs competitivas en la Alianza del Pacífico y países participantes de América del Sur, 1-40.

Defín, F. L., y Acosta, M. P. (2016). Importancia y análisis del desarrollo empresarial. Pensamiento \& Gestión, (40), 184-202.

De la Cruz, I., Morales, J., y Carrasco, G. (2006). Construcción de un instrumento de evaluación de capacidades en la empresa: Una propuesta metodológica. En las memorias del $X$ Congreso Anual de la Academia de Ciencias Administrativas, A.C. (acacia). San Luis Potosí (México), 1-30.

Donawa, Z. A. y Morales, E. C. (2018). Fuerzas competitivas que moldean la estrategia en la gerencia del sector MiPyME del distrito de Santa Marta-Magdalena, Colombia. Revista EAN, (84), 97-108.
Esparza, R. S. A., y Martínez, A. J. A. (2019). El Impacto de las Capacidades Generales y de Emprendimiento en el Valor de las Ventas de Mipymes Michoacanas. Revista de Gestión Empresarial y Sustentabilidad, 4(1), 57-73.

Gamboa-Poveda, J. E., Armijo-Borja, G. M., Pluas-Salazar, R. M., y Tovar-Arcos, G. R. (2019). La logística como instrumentos de gestión empresarial. Revista Científica FIPCAEC (Fomento de la investigación y publicación en Ciencias Administrativas, Económicas y Contables). Polo de Capacitación, Investigación y Publicación, 4(1 Especial), 250-265.

Hernández, A., Marulanda, C. E., y López, M. (2014). Análisis de capacidades de gestión del conocimiento para la competitividad de pymes en Colombia. Información tecnológica, 25(2), 111-122.

Islas, J. A. y Palomo , M. A. (2018). Impacto de las capacidades de las TIC que contribuyen a mejorar el desempeño de las MIPyMES. Innovaciones de Negocios, 16(31), 118-142.

Juárez, F. (2018). The growth of companies as a function of total assets. WSEAS Transactions on Business and Economics, 15(29), 301-310.

León-Mendoza, J. C. (2019). Emprendimiento empresarial y crecimiento económico en Perú. Estudios Gerenciales, 35(153), 429-439.

López-Salazar, A., Molina-Sánchez, R., y Gómez-Hernández, D. (2018). Enfoque estratégico, orientación emprendedora y experiencia: factores que impulsan el desarrollo de capacidades tecnológicas en el sector automotriz. Small Business International Review, 2(1), 4357.

Marín, A. (2017). Relación entre la capacidad de absorción y el desempeño innovador: Estudio multicaso en un grupo de empresas vinculadas al Centro de Investigación y Desarrollo Tecnológico del Sector Eléctrico (CIDET). Libros Universidad Nacional Abierta ya Distancia, 423-473.

Melgarejo, Z., Ciro, D., y Simón Elorz, K. (2019). Determinantes clave en el debate teórico sobre crecimiento empresarial. Criterio Libre, 18 (31), 275-296. 
Mojica, E. P. y Martínez, M. del C. (2017). Orientación al mercado, innovación y capacidades competitivas, determiantes del desempeño de las Pymes del estado de Aguascalientes. Revista Iberoamericana de Contaduría, Economía y Administración, 6(18), 1-39.

Nuryakin, Didiek, V., y Budi, M. (2018). Mediating effect of value creation in the relationship between relational capabilities on business performance. Contaduría y Administración, 63(1), $1-21$.

Ornelas, R. (2017). Hacia una economía política de la competencia. La empresa transnacional. Problemas del desarrollo, 48(189), 9-32.
Ponce-Espinosa, G., Espinoza, D. L., Ríos-Zaruma, J., y Tapia, K. G. (2017). Capacidades organizacionales generadoras de valor: análisis del sector industrial. RETOS. Revista de Ciencias de la Administración y Economía, 7(13), 271290.

Sistema de Información Empresarial Mexicano (2019). Recuperado de: https://www. gob.mx/tuempresa/es/articulos/sistema-de-informacion-empresarial-mexicano-siem-149165? idiom=es 
